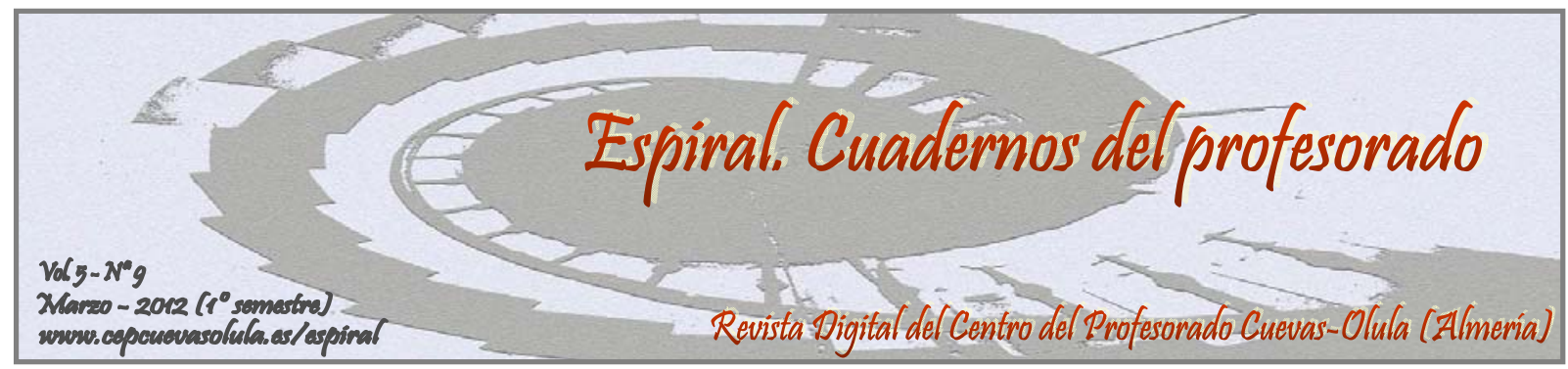

\title{
EL BLOG COMO INSTRUMENTO DE ENSEÑANZA-APRENDIZAJE DE IDIOMAS
}

\author{
BLOG AS A TEACHING AND LEARNING OF LANGUAGES \\ Margarita Pinos Pérez \\ Escuela Oficial de Idiomas, Huércal-Overa, Almería, España
}

\begin{abstract}
RESUMEN: Las actuales corrientes pedagógicas proponen un enfoque constructivista (teorías de Vigotsky (Ivic, 1994)) y un aprendizaje colaborativo (Ausubel y Novak (Daley, 2010)); por lo que las analogías con la Web 2.0 son evidentes, pues ésta se basa en la creación de conocimiento compartido. El término Web 2.0 está asociado con aplicaciones web para compartir información y una de las herramientas utilizadas es el blog. Se pretende familiarizar al profesorado y al alumnado implicado con esta herramienta. Respecto al aprendizaje de la lengua en sí misma, se persigue una mejora de las cuatro destrezas comunicativas. Se utiliza la herramienta en el aula, pero se fomenta el aprendizaje autónomo, método especialmente útil en este tipo de centros. No se utiliza el blog como una simple bitácora, sino que se transforma en un medio para crear una interacción entre el alumnado, promoviendo así el aprendizaje colaborativo. Se propone el uso de los blogs como un elemento adicional a las clases, bien como un refuerzo o como profundización y ampliación de conocimientos lingüísticos y culturales, pero nunca como un sustituto de éstas.
\end{abstract}

Palabras clave: blog, enseñanza idiomas, destrezas, cooperación, autonomía del aprendizaje.

ABSTRACT: The current educational theories suggest a constructivist model (Vigotsky's theories (Ivic, 1994)) and a collaborative learning (Ausebel and Novak (Daley, 2010)); so the analogies with the Web 2.0 are obvious, as its concepts relies on the creation of shared knowledge. The term Web 2.0 is related to applications to share information and one of the main tools that can be used is the blog. One of the main objectives is that both teachers and students familiarize themselves with this tool. Regarding the learning of the foreign language itself, the main goal is that students improve the four communicative skills. This tool is used in the classroom and also employed to promote autonomous learning, which is very useful in this kind of schools. The blog is not used as a mere log, but is converted into a way to create interaction among students, promoting cooperative learning. The blog would be seen as an additional item to the lesson, either as a reinforcement or deepening and widening tool for language and cultural knowledge, but never as a substitute to lessons.

Key words: blog, teaching languages, skills, cooperation, autonomous learning.

Pinos Pérez, M. (2012). El blog como instrumento de enseñanza-aprendizaje de idiomas. Espiral. Cuadernos del Profesorado, 5(9), 26-36 Disponible en: http://www.cepcuevasolula.es/espiral.

Fecha de recepción: 31/08/2011

Fecha de aceptación: 13/12/2011
Enviar correspondencia a: margarita_eeooii@hotmail.com 


\section{1.- INTRODUCCIÓN}

En la actualidad se puede afirmar que estamos inmersos en una sociedad de la información, la comunicación y el conocimiento, y la importancia que han adquirido los medios es tal que, sin duda alguna, la práctica educativa tiene que aliarse con éstos para lograr la consecución de los objetivos de una manera más eficaz y cercana a todo el alumnado.

Las teorías tradicionales sobre el aprendizaje se han centrado en estudiar la interacción entre el estudiante y su entorno. Sin embargo, la introducción de las tecnologías de la información y la comunicación (TIC) ha propiciado que, en un principio, se estudiasen los procesos individuales de aprendizaje; no obstante, el desarrollo de estas tecnologías ha incrementado el trabajo colaborativo y, por tanto, los estudios se centran actualmente en los procesos de aprendizaje colaborativos. Respecto al aprendizaje colaborativo asistido por ordenador, Gros (2004) expone:

El aprendizaje colaborativo mediado por ordenador supone una posible vía de utilización que ofrece varias ventajas y que expresa dos ideas importantes. En primer lugar, la idea de aprender de forma colaborativa, con otros, en grupo. No se contempla al aprendiz como persona aislada sino en interacción con los demás. Se parte de la idea de la colaboración y, por consiguiente, compartir objetivos y distribuir responsabilidades son formas deseables de aprendizaje. Además, se enfatiza el papel del ordenador como elemento mediador que apoya este proceso.

Este CSCL (del inglés Computer Supported Collaborative Learning) es un paradigma cada vez más usado en ambientes educativos; no obstante, tal y como reflejan Stahl, Koshmann y Suthers (2006), esta perspectiva presenta algunos inconvenientes. En primer lugar, no basta con subir contenido, sino que además éste debe incluir recursos relevantes para los estudiantes que estén dentro de un contexto interactivo y que les motiven. En segundo lugar, el profesor no actuará como mero observador, sino que deberá motivar a los estudiantes a través de mecanismos de interacción, por lo que su esfuerzo aumentará significativamente. En tercer lugar, los estudiantes no deberán trabajar aisladamente con materiales incluidos, sino que se fomenta la colaboración entre ellos. Su aprendizaje aumenta mediante la colaboración con sus compañeros formulando preguntas, planeando actividades conjuntas, etc.

Las herramientas de la Web 2.0, blogs y wikis, se pueden utilizar para facilitar el CSCL. Los blogs ofrecen un amplio abanico de posibilidades de uso en procesos educativos. Según Vidal (2006), los blogs suponen una manera de hacer las TIC invisibles, es decir, no se dan, sino que se utilizan. Además, afirma que las TIC no son un fin en sí mismas, sino un soporte para desarrollar contenidos.

A este respecto, Marquès (2000) establece las principales funciones de las TIC en los entornos educativos; la lista que recoge es mucho más amplia, pero se incluyen las que hacen referencia directa a este estudio, y se comentan aspectos relativos a éste:

- Medio de expresión: escribir, dibujar, presentaciones, webs. En el caso de los blogs, se trata de un medio de expresión donde no es exclusivamente el profesorado el que realiza aportaciones, sino que el alumnado también contribuye.

- Canal de comunicación, pues que la información es la materia prima para la construcción de conocimientos, La comunicación puede ser tanto presencial mediante la que el alumnado puede participar más en clase (por ejemplo, mediante la pizarra digital), como de forma virtual mediante mensajería, fotos, weblogs, wikis, plataformas, que facilitarían trabajos en colaboración, intercambios, tutorías, compartir, poner en común, negociar significados, informar. En este estudio se recogen ambos canales de comunicación, tanto presencial, puesto que se utilizará el blog como apoyo con material para realizar actividades dentro del propio aula, así como la forma virtual, que será la que esté presente en un mayor número de actividades.

- Instrumento para procesar la información. Hay que procesar la información para construir nuevos conocimientos-aprendizajes. Los blogs suponen una nueva forma de procesar in- 
formación y, consecuentemente, de aprender. Para una gran parte del alumnado puede suponer un nuevo método de aprendizaje que poco a poco valorarán.

- Generador / Espacio de nuevos escenarios formativos, ya que multiplican las oportunidades de aprendizaje contribuyendo a la formación continua en todo momento y lugar. Sin duda alguna, esta herramienta aquí presentada claramente brinda una oportunidad de aprendizaje en cualquier momento y en cualquier lugar. Aspecto que resulta sumamente útil para el tipo de alumnado que se ve implicado en este estudio, puesto que tienen responsabilidades que pueden impedirles desplazarse físicamente al centro.

- Las actividades suelen resultar motivadoras (imágenes, vídeo, sonido, interactividad...), y la motivación es uno de los motores del aprendizaje. En este estudio se comprueba que el alumnado se motiva al encontrarse con situaciones reales a través de discursos, conversaciones, canciones, etc., acelerando así su proceso de aprendizaje.

- Pueden facilitar la labor docente: más recursos para el tratamiento de la diversidad, facilidades para el seguimiento y evaluación (materiales autocorrectivos, plataformas...), tutorías y contacto con las familias... En cuanto a la diversidad, queda claro que el alumnado involucrado en este estudio tienes unas características muy diferentes, en lo que se refiere a los estilos de aprendizaje, al ritmo con el que cada persona adquiere las destrezas comunicativas y, obviamente, a los intereses y necesidades que cada persona tiene respecto al aprendizaje de la lengua. Los blogs sin duda alguna permiten ampliar las tareas e incluir una diversidad mayor de la que podemos utilizar en el propio aula.

- Suponen el aprendizaje de nuevos conocimientos y competencias que inciden en el desarrollo cognitivo y son necesarias para desenvolverse en la actual Sociedad de la Información. Por tanto, queda claro que el manejo de las nuevas tecnologías es una asignatura obligada en la sociedad actual, por lo que se le brinda al alumnado la posibilidad de profundizar en su manejo.

El proyecto desarrollado se centra en los blogs de aula donde el profesor publica tareas y actividades educativas para fomentar la escritura de bitácoras como herramienta para publicación de contenidos, fomentar el trabajo en colaboración sumando las aportaciones individuales, capacitar la escritura para expresar opinión, experiencias, fomentar la creatividad y propiciar el debate.

Los docentes pueden utilizar los blogs para acercarse a los estudiantes de una forma nueva y atractiva sin tener que limitar su interacción exclusivamente al aula. Se pueden publicar materiales de manera inmediata y permitir el acceso a información o a recursos necesarios para realizar proyectos y actividades de aula, optimizando así el tiempo de clase. Además, ofrece a los estudiantes la posibilidad de mejorar los contenidos académicos enriqueciéndolos con diversos elementos, como vídeos, sonidos, imágenes, juegos, etc.

Vidal (2006) afirma que los blogs ofrecen momentos y situaciones para que todos los alumnos y alumnas adquieran cotas de protagonismo. Por eso mismo, y promoviendo la motivación, en este estudio se hace al alumnado partícipe activo del proceso educativo, ya que se le puede estimular a escribir, intercambiar ideas, trabajar en equipo, diseñar, visualizar de manera instantánea lo que produce, etc.

Los trabajos colaborativos se han venido posicionando como una buena estrategia educativa. Por tanto, con los blogs se supera la dificultad de que tengan que estar todos los miembros de un grupo en un mismo espacio físico, pues ofrecen un espacio virtual, independiente del emplazamiento. Esto posibilita al docente, actuando como facilitador del aprendizaje, guiar constantemente a los estudiantes.

El término 'e-moderating' fue empleado por primera vez por Salmon (2000) para describir las funciones y destrezas que el profesor o formador online tiene que adquirir para fomentar el aprendizaje online. La función del docente se ve ampliada con respecto a la formación meramente presencial, puesto que es necesario que se adecúe a los nuevos entornos de aprendizaje. Está claro que los docentes deben poseer cierto bagaje del uso de Internet que poco a poco irá aumentando con la 
progresión del proyecto. Según explican González y Salmon (2002), existen ciertos periodos en la formación del 'e-moderator'. En primer lugar, se debe ser consciente de que al aumentar el bienestar de los participantes online, éstos aumentan sus contribuciones. Por tanto, es fundamental no desesperarse al principio, puesto que los estudiantes van participando a medida que van mostrando confianza, y esa confianza viene dada por el propio profesor y los compañeros. En segundo lugar, se debe tener en cuenta que el diseño de actividades online y la interacción son tan importantes como los aspectos más sofisticados del diseño y presentación de los contenidos. Tanto es así, que los estudiantes mediante una simple pregunta que les haga reflexionar y crear un debate, se muestran más participativos que si se les formula otro tipo de pregunta menos polémica presentada con muchos más medios, aunque lo ideal es unir los dos aspectos. En tercer lugar, la clara percepción de que el papel del 'e-moderator', que abarca mucho más que las tareas de un mero facilitador o contestador de preguntas, decidirá el éxito o fracaso de la experiencia de los estudiantes. Bien es cierto, que el hecho de que sea la primera vez que el alumnado utiliza un blog para apoyar su proceso de aprendizaje de un idioma, pueda ser el condicionante para que sigan con este método más adelante o, por el contrario, lo abandonen; consecuentemente tienen que sentir que están aprendiendo y consiguen mejorar. En cuarto lugar, el reconocimiento de que existe considerable evidencia de que los participantes se vuelven más independientes y responsables de su propio desarrollo a medida que avanzan por los estadios del modelo, tanto en situaciones de aprendizaje estructurado como informal. Este último punto que resaltan estos autores coincide con uno de los objetivos del MCER (Marco Común Europeo de Referencia para las Lenguas), concretamente al conocido como «aprender a aprender», que sin duda alguna es uno de los objetivos perseguidos para nuestros estudiantes que tarde o temprano terminarán sus estudios y deberán seguir formándose por sí mismos:

«Aprender a aprender» es la habilidad para iniciar el aprendizaje y persistir en él, para organizar su propio aprendizaje y gestionar el tiempo y la información eficazmente, ya sea individualmente o en grupos. Esta competencia conlleva ser consciente del propio proceso de aprendizaje y de las necesidades de aprendizaje de cada uno, determinar las oportunidades disponibles y ser capaz de superar los obstáculos con el fin de culminar el aprendizaje con éxito. Dicha competencia significa adquirir, procesar y asimilar nuevos conocimientos y capacidades, así como buscar orientaciones y hacer uso de ellas. El hecho de «aprender a aprender» hace que los alumnos se apoyen en experiencias vitales $y$ de aprendizaje anteriores con el fin de utilizar y aplicar los nuevos conocimientos y capacidades en muy diversos contextos, como los de la vida privada y profesional y la educación y formación. La motivación y la confianza son cruciales para la adquisición de esta competencia. (Parlamento y Consejo Europeo, 2006, p. 7)

El concepto de aprendizaje autónomo lo define Lileya (2004) como la facultad que le permite al estudiante tomar decisiones que le conduzcan a regular su propio aprendizaje en función de una determinada meta y de un contexto o condiciones específicas de aprendizaje.

No sólo es relevante el concepto de aprendizaje autónomo, sino también el de aprendizaje colaborativo. Lave y Wenger (1991) nos introducen en el concepto de legítima participación periférica cuando hablan de las relaciones entre los novatos y los más expertos, y de las identidades, artefactos, tareas y comunidades de práctica y conocimiento. Bien es sabido que el aprendizaje no siempre requiere una instrucción explícita, sino que se puede aprender de otras muchas formas, bien mediante la observación, implicación en diversos procesos o escucha.

Tras analizar los conceptos principales que atañen a este estudio, se detallan los objetivos planteados por el profesorado implicado:

1. Familiarizar a todos los miembros con el uso de los blogs como herramienta para mejorar la práctica docente.

2. Compartir experiencias entre los departamentos de inglés y francés de la escuela, así como actividades que se puedan llevar a cabo en ambos idiomas.

3. Promover el uso de las nuevas tecnologías entre el alumnado, haciéndoles ver su utilidad en el aprendizaje de idiomas y desarrollando a la vez su competencia tecnológica. 
4. Analizar la forma de mejorar las destrezas de comprensión escrita, comprensión oral, expresión escrita e, incluso, expresión oral de los estudiantes mediante el uso de esta nueva herramienta, tanto en los blogs de cada docente como en el blog común de la escuela.

5. Suscitar la participación activa del alumnado en el blog. Supone un aspecto novedoso, ya que los blogs que se han consultado, creados por el profesorado para la enseñanza de idiomas, no recogen esta participación. Se pretende que en todo momento esté controlada por los propios docentes.

6. Promover en el alumnado la capacidad de aprendizaje autónomo, especialmente útil en el caso de las Escuelas Oficiales de Idiomas, puesto que la diversidad del alumnado supone que existan estudiantes con intereses y formas de aprendizaje diferentes. Así, el alumnado puede elegir entre los temas publicados aquellos que más le interese, así como reforzar aquellas destrezas en las que encuentren mayor dificultad, siendo ellos en todo caso los que se decidan por unas actividades u otras.

7. Fomentar el aprendizaje colaborativo y el trabajo en grupo.

8. Promover el debate a través de los comentarios entre el alumnado y el profesorado.

9. Crear una herramienta de apoyo para el alumnado que se vea obligado a ausentarse del aula por algún motivo, así como poder ponerse en contacto con sus compañeros.

\section{2.- DESCRIPCIÓN DEL TRABAJO REALIZADO}

En el especial de EducaWeb "Las TIC en las aulas", se afirma que la progresiva introducción de las TIC en el contexto educativo supone una redefinición de las funciones docentes y del proceso de enseñanza-aprendizaje. Todo ello implica que estas técnicas conllevan un cambio en la forma de impartir clase, puesto que el profesor no dará una clase magistral y el alumnado memorizará los conocimientos, sino que habrá un trabajo colaborativo, participación por parte del alumnado y un nuevo rol para el docente. Teniendo en cuenta estas ideas, el proyecto que nos ocupa se puede estructurar en tres fases (fase inicial, intermedia y final), algunas de ellas con una duración más extensa que otras por la elaboración de materiales y familiarización con el proyecto.

\section{Fase inicial}

La primera fase consistió en la creación por parte de cada uno de los miembros del proyecto de un blog personal, o bien en la ampliación de uno previamente creado en otros cursos con un fin común al de este proyecto. En dos reuniones presenciales se ofrecieron las líneas generales para la creación del blog en blogger.com, aunque estas directrices son extrapolables a otras plataformas. Además, se subió a la plataforma 'Colabora' de la Junta de Andalucía un tutorial de Valero (2010) donde se recogen todos los pasos que se deben seguir de forma pormenorizada, así como algunas ampliaciones.

Una vez creado el blog, se procedió a subir material de refuerzo o extensión para el alumnado de los distintos niveles. Cada docente se centró en los cursos que estaba impartiendo. Se propuso crear etiquetas para las diferentes entradas de los cursos, quedando así organizadas para el alumnado interesado. Existe un objetivo claro consistente en mejorar las distintas destrezas, por lo que, adicionalmente, se incluyeron enlaces a otros sitios webs enfocados a alguna destreza concreta, enlaces a diccionarios, noticias, otros blogs de interés, etc. Con objeto de facilitar al alumnado el uso del blog, estos enlaces se clasificaron bajo etiquetas con el título de cada una de las destrezas.

En el aula se dio a conocer el blog al alumnado, se les enseñó el manejo de esta herramienta, de forma que pudiesen utilizarla por su cuenta, además de orientarles sobre el enfoque que se le estaba dando desde este proyecto. Conforme se fueron publicando actividades o enlaces, se hacía referencia en clase, aunque lo que se pretendía era que poco a poco fuesen ellos los que accediesen por su cuenta para comprobar si había novedades y practicar individualmente de forma autónoma.

La compresión escrita se ha trabajado mediante la lectura de textos adaptados a los conocimientos de cada grupo con preguntas sobre estos textos. A algunas de estas actividades se les ha añadido a posteriori un enlace con las soluciones correspondientes. Por otra parte, también se ha trabajado esta destreza mediante la lectura de noticias aunque no estuviesen adaptadas al nivel específico de cada grupo, con lo que se han adquirido las destrezas necesarias para entender un texto 
en su conjunto.

Para mejorar la comprensión oral se incluyeron vídeos, canciones, trailers de películas, etc. En algunas de estas entradas se han añadido actividades que el alumnado realizó individualmente.

Respecto a la expresión e interacción escrita, tal y como se menciona en la introducción, se propuso que el alumnado realizase comentarios en las diferentes entradas del blog. Así se han creado algunas entradas específicas con este fin, con preguntas sobre temas tratados en clase o temas de actualidad que despertasen su curiosidad y sobre los que quisieran dar su opinión.

En algunas ocasiones, para practicar la destreza de expresión e interacción oral, se ha realizado alguna actividad en clase utilizando material del blog, bien mediante un vídeo, texto o imágenes que suscitasen debate por parte del alumnado, consiguiendo una interacción oral entre ellos.

A modo de ejemplo, se puede ver una de las entradas de unos de los blogs en la figura 1.

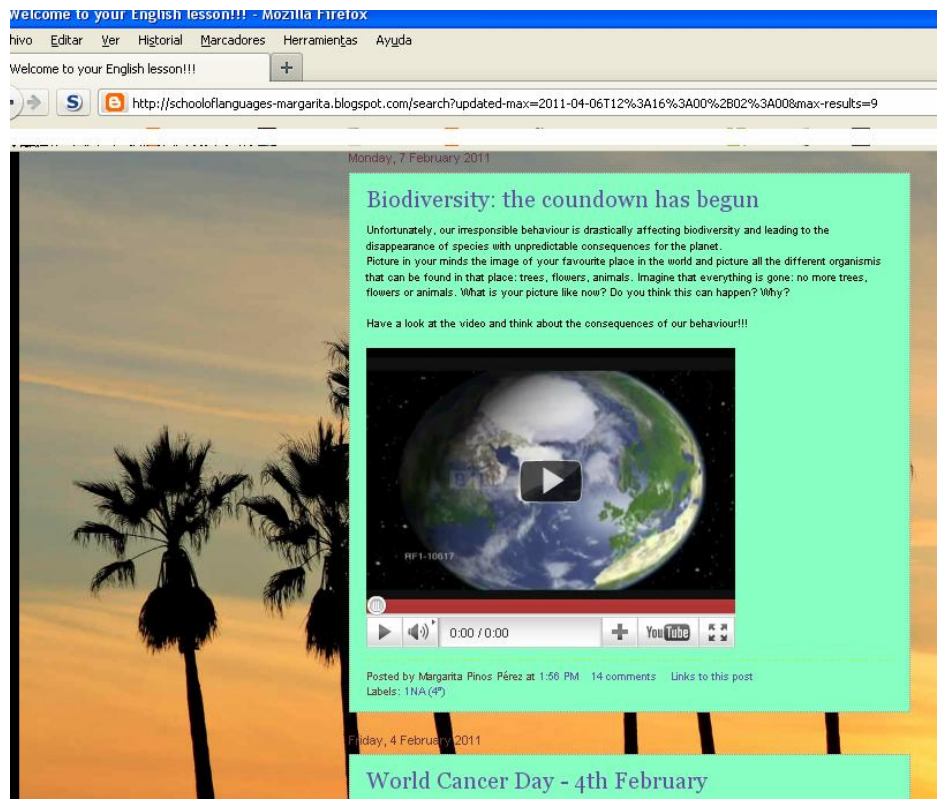

Figura 1.- Actividad de expresión e interacción escrita donde el alumnado reflexiona sobre la repercusión de sus actos en el medio ambiente.

\section{Fase intermedia}

Una vez creados los blogs individuales y con el alumnado familiarizado con esta nueva herramienta, se continuó con la creación del blog común para la escuela siguiendo el mismo procedimiento de creación de los individuales. En el momento en que se establecieron los objetivos de este proyecto, se pretendía que tanto los docentes como el alumnado fuesen administradores del mismo, y ambos colectivos pudieran publicar libremente, que es lo que se conoce como blog colectivo. Sin embargo, se concluyó que ésta era una opción inviable, puesto que en ese caso se podría publicar material no adecuado e, incluso, restringir el acceso al blog por parte de los docentes. Por ello, se optó por crear un blog común que funcionase como tablón de anuncios sobre las actividades que se organicen en la escuela (o fuera de ella) relacionadas con los idiomas, las actividades extraescolares, concursos, publicación de exámenes, fechas relevantes, etc. Dado que uno de los objetivos de este proyecto era que el alumnado participase activamente en este blog común, se pensó en una alternativa, así que se les informó de que en caso de que deseasen compartir alguna experiencia con sus compañeros, no dudasen en facilitar el material correspondiente, que se subiría sin ningún problema.

En este blog se incluyeron enlaces a los blogs de cada uno de los docentes implicados en este proyecto. Se ha comprobado que esta herramienta les ha resultado de utilidad a los estudiantes que podían acceder a dichos blogs directamente.

En la figura 2 se puede observar una de las entradas de este blog común. 


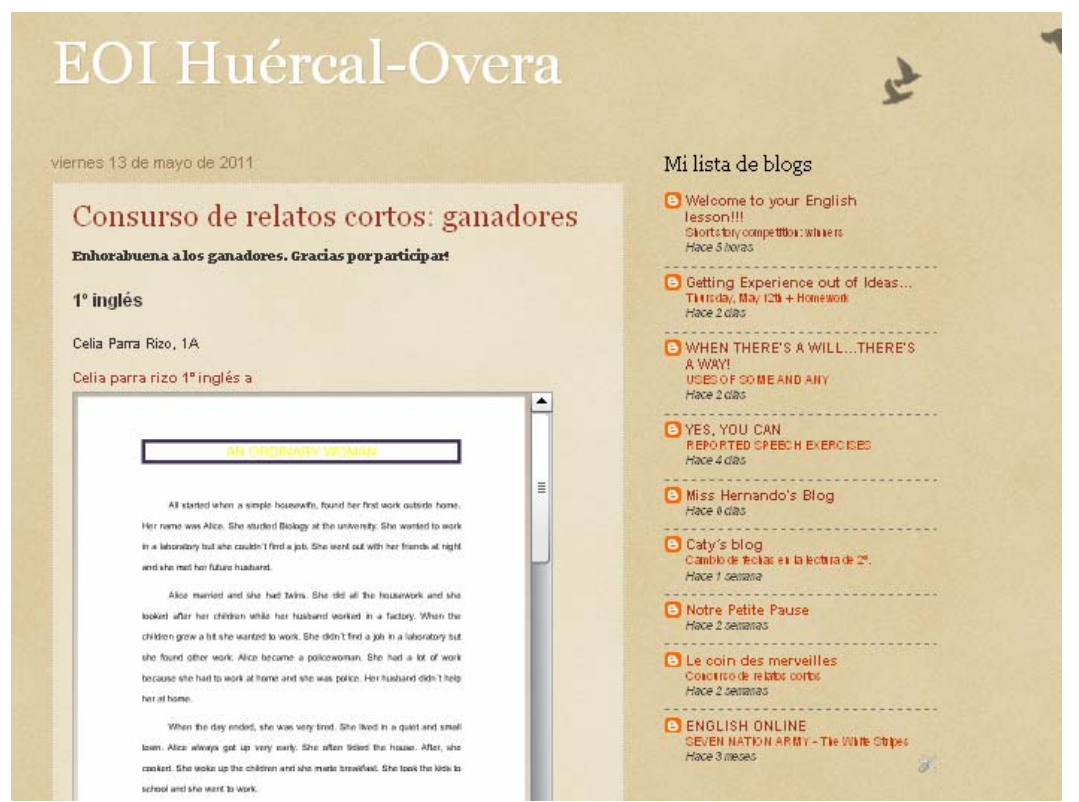

Figura 2.- Entrada de los ganadores del concurso de relatos de inglés y francés.

\section{Fase final}

Se ha seguido actualizando el blog de la escuela con entradas sobre actividades, noticias u otros eventos relacionados con la escuela o aspectos de interés para el alumnado. Además, cada docente ha incluido hipervínculos o actividades progresivas para los diferentes niveles en los blogs individuales, de forma que el alumnado siguiese practicando y mejorando las destrezas que han presentado más dificultades. Dichas actividades se han centrado principalmente en el trabajo que se estaba realizando en clase. Regularmente se ha hecho mención en clase a las novedades que se han publicado en el blog para que el alumnado que no lo visitase regularmente, así lo hiciese.

Se ha insistido al alumnado en que plasmase sus comentarios en las entradas que ya se habían publicado, y en que fuesen críticos en las respuestas a los comentarios de sus compañeros.

\section{3.- RESULTADOS Y REPERCUSIONES EN LA COMUNIDAD EDUCATIVA}

Se analizan los resultados obtenidos teniendo en cuenta los objetivos planteados en este proyecto.

El objetivo de partida consiste en familiarizar al profesorado con esta herramienta. Se debe destacar que todos los docentes crearon con éxito su blog de aula y fue dado a conocer al alumnado, que se mostró en todo momento muy receptivo, a pesar de que para algunos se trataba de una herramienta totalmente nueva. Si bien en este punto cabe destacar, como afirman Kumar y Tammelin (2008), que si un docente no ha desarrollado nunca antes un sitio web para su curso, le vendría muy bien ver ejemplos de cómo son utilizados para complementar cursos de lengua extranjera. Por lo tanto, el profesorado que empieza a usar las TIC se beneficia al conocer otros modelos y buenas prácticas, así se anima a reflexionar sobre qué tecnologías trabajar en un contexto, con un alumnado y con un plan de estudios concreto. En este estudio concreto, y al trabajar unidos dos departamentos didácticos, se han podido compartir las experiencias realizadas entre los docentes implicados.

Además de familiarizar al profesorado con esta herramienta, se ha conseguido impulsar su uso entre los estudiantes, que han visto su utilidad según ha ido avanzando el proyecto. Conviene destacar que la mayoría del alumnado ha accedido a los blogs de forma regular, pero que debido a su diversidad, algunos estudiantes no cuentan con los conocimientos básicos de informática necesarios. Este factor no les ha supuesto ninguna barrera, ya que han mostrado un interés especial y han pedido ayuda a los docentes y a sus compañeros para tener acceso a las actividades.

En las sucesivas pruebas trimestrales se pudo confirmar que las destrezas del alumnado se 
vieron reforzadas, ya que cada uno era consciente de sus carencias y fueron éstas las más trabajadas en los blogs. Se pudo comprobar que el alumnado estuvo trabajando por su cuenta, gracias a los contadores de visitas colocados en los respectivos blogs. Como afirma Carretero (2005), somos conscientes de que el trabajo del alumno debe ser guiado, siendo necesario seleccionar o elaborar materiales que les servirán a los estudiantes para progresar en el inglés. A este respecto, Cabero (2007) hace una reflexión sobre el hecho de que aunque las TIC alcancen un gran impacto, no significa que desde un punto de vista cualitativo ese mayor acceso repercuta sobre la calidad de los productos que se consiga. De forma que lo importante no es cómo nos llega la información, sino qué hacemos con ella y cómo llegamos a procesarla. Carretero (2005) menciona que gracias a la combinación de los recursos tecnológicos, pedagógicos y humanos se consigue en mayor medida responder a los intereses y necesidades personales de cada individuo. Así se consigue que el alumnado trabaje a su propio ritmo, de forma que avanza según su grado real de asimilación y progreso. Además se tiene en cuenta el aprendizaje significativo, que requiere la creación de un conflicto cognitivo para que surja una motivación para el aprendizaje; los medios y las tecnologías provocan a menudo este tipo de actitudes.

Se valora positivamente el hecho de que se hayan realizado comentarios por parte del alumnado cuando se ha propuesto un tema controvertido para que opinaran sobre él. Cada docente ha gestionado dichos comentarios según ha creído más conveniente, bien corrigiéndolos para que sean gramatical y léxicamente correctos previamente a su publicación, bien publicándolos tal y como fueron presentados con un comentario posterior en el propio blog o a título individual. Adicionalmente, cabe destacar que se ha propuesto al alumnado que indique su curso al realizar comentarios en el blog común de la escuela para que así fuese posible gestionarlos adecuadamente. A este respecto, Boza y Toscano (2011), en su decálogo para planificar buenas prácticas docentes con tecnologías, mencionan que el alumnado debe aprender "haciendo cosas" con la tecnología, no siendo simples receptores pasivos. Gracias a los comentarios realizados también se ha cumplido con el objetivo inicial de promover el debate, puesto que los alumnos han ido haciendo comentarios a los de sus compañeros y basándose en ellos, promoviendo a su vez el aprendizaje colaborativo.

De acuerdo con Cabero (2006), las posibilidades que nos ofrecen las tecnologías no son sólo cuantitativas, sino también cualitativas, y esta flexibilidad nos permite adaptar la información en función de las Inteligencias Múltiples propuestas por Howard Gardner (Amarís, 2002). Se promueve así el aprendizaje autónomo, ya que todos los alumnos pueden aprender según su mejor forma de aprendizaje. Se comprueba que, a medida que ha ido avanzando el curso, la aceptación por parte del alumnado al uso de los blogs se ha visto incrementada, sobre todo de los individuales de cada docente. Dicha aceptación también queda patente en el hecho de que el alumnado haya preguntado regularmente si se iba a subir al blog cierto material trabajado en clase, o si iba a poder practicar más sobre ciertos aspectos a través de esta herramienta. Este interés ha animado al profesorado implicado a seguir publicando material, ya que ha sido evidente el aprovechamiento por parte del alumnado involucrado.

Según Castaño (2008), entre las aportaciones que hace la web 2.0 al mundo de la educación encontramos aprender con y de otros usuarios, compartiendo conocimiento; también destaca el efecto red, fundamentalmente del trabajo individual a la cooperación entre iguales. Además Kumar y Tammelin (2008) destacan que es muy común que los estudiantes de lenguas se sientan aislados de su docente y de sus compañeros/as cuanto utilizan las TICs, mientras que en otros casos, el alumnado que vacila en hablar con sus compañeros/as se siente más cómodo escribiendo sus opiniones en Internet. De esto se puede deducir que, en función del alumnado, es factible potenciar el aprendizaje colaborativo o el aprendizaje autónomo. Con este esquema se intentan promover ambas vertientes, como así lo resaltan Boza y Toscano (2011) al mencionar que las TIC deben ser utilizadas tanto para el trabajo individual de cada alumno como para el desarrollo de procesos de aprendizaje colaborativo. Además, en el caso del blog común de la escuela se propuso a los estudiantes que facilitasen cualquier material que viesen interesante y útil para compartir. 


\section{4.- CONCLUSIONES}

Se ha podido comprobar que a pesar de que los recursos electrónicos puedan resultar superficiales, el uso de blogs como herramienta de apoyo en la docencia de idiomas resulta fructífera, tanto en la mejora de las carencias del alumnado en ciertas destrezas comunicativas, como en avivar su interés en el aula, dado que pueden avanzar más rápidamente en su aprendizaje y afianzar los conocimientos ya adquiridos.

Villalba (1999) recoge los enfoques comunicativos presentados por Canale y Swain en el campo de la didáctica de las lenguas. Esta perspectiva confirma el interés por la nueva tecnología, puesto que se pone el énfasis en la comunicación. Tal y como afirma Carretero (2005), apostamos por seguir la propuesta del enfoque comunicativo que se nutre de las concepciones del modelo de investigación de acción, de la pedagogía crítica y de la adecuación a un espacio y una realidad cambiante.

El Ministerio de Educación y Ciencia (2002) hace referencia a las competencias básicas en el aprendizaje de lenguas. Así podemos afirmar que gracias a este proyecto, se puede hacer que los estudiantes adquieran competencias básicas tan esenciales en ámbitos como son la comunicación lingüística, el conocimiento y la interacción con el mundo físico y natural, el aprendizaje continuado de forma autónoma, así como competencias digitales y sociales.

Es por ello que se continuarán empleando los blogs creados en este proyecto como pilares fundamentales en la mejora de la comunicación profesor-alumno, la cual redunda en una experiencia docente más efectiva. Nuestro objetivo es superar la barrera de la perspectiva ofrecida al alumnado de los contenidos de una forma tradicional, acercándolos de esta forma al tratamiento trasversal y la integración de la tecnología, proporcionando así una forma más crítica y lúdica de aprendizaje que rentabilice las cualidades que nos proporcionan estas herramientas para lograr un aprendizaje más eficaz.

Cabero (2007) destaca que los profesores y formadores no van a ser reemplazados por las tecnologías por muy potentes y sofisticadas que sean, aunque tendrán que cambiar los roles y actividades que actualmente desempeñan.

A continuación, y a modo de conclusión, se recoge una selección de los blogs creados durante este proyecto:

Blogs de los docentes implicados en el proyecto y blog común:

Blog de la escuela: http://eoihuercal-overa.blogspot.com/

Caty's blog: http://catyenglish.blogspot.com/

English online: http://eoienglishonline.blogspot.com/

Getting experience out of ideas...: http://lfas-eoi.blogspot.com/

Le coin des merveilles: http://lecoindemeseleves.blogspot.com/

Miss Hernando's blog: http://misshernando.blogspot.com/

Notre Petite Pause: http://notrepetitepause.blogspot.com/

Welcome to your English lesson: http://schooloflanguages-margarita.blogspot.com/

When there's a will..., there's a way: http://mavimoya.blogspot.com/

Yes, you can: http://encarnisblog.blogspot.com/

Para concluir este estudio, merece la pena recordar las palabras del Premio Nobel de Literatura de 1990, Octavio Paz, respecto al uso de tecnología, por la que hoy en día tanto se aboga desde muchos ámbitos educativos:

Creo que estamos condenados a ser modernos. No podemos prescindir de la ciencia y de la técnica.

El problema consiste en adecuar la tecnología a las necesidades humanas y no a la inversa como ha ocurrido hasta ahora. 


\section{5.- REFERENCIAS}

Amarís Macías, M. (2002). Las múltiples inteligencias. Psicología desde el Caribe [en línea]. Colombia. (27-38) Disponible en: http://redalyc.uaemex.mx/pdf/213/21301003.pdf [Consulta: 2011, 20 de noviembre].

Boza Carreño, A., y Toscano Cruz, M. (2011). Buenas prácticas en integración de las TIC en educación en Andalucía: 2 estudios de caso. Ponencia: VI Congreso Virtual de AIDIPE [en línea]. Disponible en: http://www.uv.es/aidipe/congresos/Ponencia_VIICongresoVirtual_AIDIPE.pdf [Consulta: 2011, 17 de noviembre].

Cabero Almenara, J. (2007). Las necesidades de las TIC en el ámbito educativo: oportunidades, riesgos y necesidades. Tecnología y Comunicación Educativas Año 21, 45, 4-19 [en línea]. Disponible en: http://investigacion.ilce.edu.mx/tyce/45/articulo1.pdf [Consulta: 2011, 20 de noviembre].

Carretero Ramos, A. (2005). Las TICs en el aula de inglés: un proyecto de trabajo [en línea]. Disponible en: http://www.quadernsdigitals.net/index.php?accionMenu=hemeroteca.VisualizaArticuloIU.visualiza\&art iculo id=8656 [Consulta: 2011, 22 de noviembre].

Castaño Garrido, C. (2008). Aprender con redes sociales y Web 2.0. En J. Salinas Ibáñez, Innovación Educativa y Uso de las TIC (67 - 82). Sevilla: Universidad Internacional de Sevilla [en línea]. Disponible en: http://dspace.unia.es/bitstream/10334/131/1/004tic_casta\%C3\%B1o.pdf [Consulta: 2011, 13 de noviembre].

Daley, B. (2010). Concepts maps: Practice applications in adult education and human resource development (perspectives on practice). New Horizons in Adult Education and Human Resource Development [en línea] 24. (2-4) 30-36 Disponible en: http://education.fiu.edu/newhorizons/journals/24_24/Concept\%20MapPerspective_BD24\%282-4\%29.pdf [Consulta: 2011, 18 de noviembre].

Educaweb.com. Las TIC en el aula. Educaweb.com [en línea]. Disponible en: http://ems6.net/a/?F=wmad27h953lekvar4ey3lv8dpfdr5g8vly9wkx5zk63mkjrfaqsm5zz-1436979 [Consulta: 2011, 8 de abril].

González F., y Salmón, G. (2002). La función y formación del E-moderator: clave del éxito en los nuevos entornos de aprendizaje. Online Educa, Barcelona [en línea]. Disponible en: http://www.atimod.com/presentations/download/educaspanish.doc [Consulta: 2011, 15 de junio].

González, M. (2007). TIC y enseñanza de lenguas. Cuaderno Intercultural [en línea]. Disponible en: http://www.cuadernointercultural.com/tic-y-ensenanza-de-lenguas/ [Consulta: 2011, 15 de mayo].

Gros Salvat, B. (2004). La construcción del conocimiento en red: límites y posibilidades. Teoría de la Educación: educación, cultura y sociedad de la información [en línea]. Disponible en: http://campus.usal.es/ teoriaeducacion/rev_numero_05/n5_art gros.htm [Consulta: 2011, 4 de mayo].

Ivic, I. (1994). Lev Semionovich Vygotsky. Perspectivas: revista trimestral de educación comparada [en línea] $\begin{array}{llllll}\text { Vol. XXIV, } & \text { (n. } & 3-4) & \text { 773-799. }\end{array}$ http://www.ibe.unesco.org/publications/ThinkersPdf/vygotskys.PDF [Consulta: 2011, 12 de noviembre].

Kumar, S., y Tammelin, M. (2008). Integrar las TIC en la enseñanza/aprendizaje de segundas lenguas. Viena: Johannes Kepler Universität Linz [en línea]. Disponible en: http://webh01.ua.ac.be/odlac/guides/4cGUIDES-INSTITUTIONS-ES.pdf [Consulta: 2011, 19 de noviembre].

Lave, J., y Wenger, E. (1991). Situated learning. Legitimate peripheral participation. Cambridge: Cambridge University Press.

Lileya Manrique, V. (2004) El aprendizaje autónomo en la educación a distancia. Primer Congreso Virtual Lationamericano de educación a distancia. [en línea]. Disponible en: http://www.ateneonline.net/datos/55_03_Manrique_Lileya.pdf [Consulta: 2010, 13 de junio].

Marquès Graells, P. (2000, versión 2011). Impacto de las TIC en educación: funciones y limitaciones. Departamento de Pedagoría Aplicada. Faculta de Educación (UAB) [en línea]. Disponible en: http://www.peremarques.net/siyedu.htm [Consulta: 2011, 17 noviembre].

MEC (2002). Marco común europeo de referencia para las lenguas: aprendizaje, enseñanza, evaluación. Instituto Cervantes [en línea]. Disponible http://cvc.cervantes.es/ensenanza/biblioteca_ele/marco/cvc_mer.pdf [Consulta: 2011, 28 noviembre].

Parlamento y Consejo Europeo (2006) Competencias clave para el aprendizaje permanente - un marco de referencia europeo. Diario oficial de la unión europea L 394/13 del 30 de diciembre de 2006. [en línea]. Bruselas. 
lex.europa.eu/LexUriServ/LexUriServ.do?uri=OJ:L:2006:394:0010:0018:ES:PDF [Consulta: 2011, 19 de junio].

Salmon, G. (2000) E-Moderating: The Key to Teaching and Learning Online. Londres: Kogan Page.

Stahl, G., Koschmann, T., y Suthers, D. (2006). Computer-supported collaborative learning: An historical perspective. En R. K. Sawyer (Ed.), Cambridge handbook of the learning sciences (pp. 409-426). Cambridge, UK: Cambridge University Press. [en línea]. Disponible en: http://www.cis.drexel.edu/faculty/gerry/cscl/CSCL_English.pdf [Consulta: 2011, 7 de julio].

Valero, A. (2010). Cómo crear un blog en blogger: creación, configuración, diseño y multimedia. Apuntes sobre blogs [en línea]. Disponible en: http://avalerofer.blogspot.com/2007/02/cmo-crear-un-blog-enblogger.html [Consulta: 2010, 13 de diciembre].

Vidal Uraga, I. (2006). Uso didáctico de los blogs [en línea]. Disponible en:

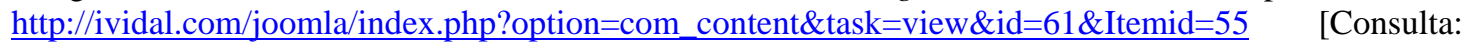
2011, 10 de noviembre].

Villalba, F., y Hernández, M. T. (1999). Concepto de lengua y teorías de aprendizaje en el enfoque comunicativo de idiomas [en línea]. Cuadernos de Bitácora, $\mathrm{n}^{\circ}$ 4. Disponible en: http://segundaslenguaseinmigracion.com/ense_anzal2/conceptodelengua.pdf (109-126) [Consulta: 2011, 14 de noviembre]. 